\title{
Cause-specific mortality and associated factors related to death after kidney and liver transplantation: a Korean nationwide study
}

\author{
Junghyun Yoon ${ }^{1}$, Younkyung Jung ${ }^{2}$, Hanjoon Kim², Boyoung Park ${ }^{1}$, Dongho Choi ${ }^{2}$ \\ ${ }^{1}$ Department of Preventive Medicine, Hanyang University College of Medicine, Seoul, Korea \\ ${ }^{2}$ Department of Surgery, Hanyang University College of Medicine, Seoul, Korea
}

Background: Although the demand for organ transplantation increased, the survival rate of transplants was insufficient compared to the general population due to the use of immunosuppressants. Few studies have been published in Korea related to death and were limited to single institutions and small cohorts. The purpose of this study was to analyze the mortality rate, cause of death, and the factors associated with death in kidney and liver transplant recipients.

Methods: Study population was the recipient of kidney and liver transplants from National Health Insurance Service database between 2006 and 2017. A total of 17,446 kidney transplants and 11,590 liver transplants were identified, and the transplants were linked to data on the cause of death from Statistics Korea. The cause of death of kidney and liver transplantation were classified into renal and non-renal, hepatic and non-hepatic, respectively, based on International Classification of Diseases (ICD).

Results: In kidney transplants, renal death was $35 \%-42 \%$, of which chronic kidney disease is the main cause. The proportion of non-renal death was highest in the order of malignancy and infection. In liver transplants, hepatic death accounted for $65 \%-67 \%$. Among them, hepatic malignancy was high in 5-year mortality and death from cirrhosis or alcoholic liver disease were higher in overall mortality. Infection was the leading cause of death in non-hepatic, with $22 \%$ at 5 -year mortality and $17 \%$ in overall mortality. In the underlying etiology, the risk of death was high in kidney transplants with diabetes and liver transplants with malignancy. Conclusions: After kidney and liver transplantation, the most common cases of death were each primary disease, and there were differences in death patterns of malignancy, infections, and cardiovascular diseases according to organ characteristics. Since the distribution of causes of death varies depending on the period after transplantation, the survival rates can be improved through appropriate interventions at each time.

Corresponding author: Dongho Choi

E-mail: crane87@hanyang.ac.kr

\footnotetext{
(C) The Korean Society for Transplantation

This is an Open Access article distributed under the terms of the Creative Commons Attribution Non-Commercial License (http://creativecommons.org/licenses/by-nc/4.0/) which permits unrestricted non-commercial use, distribution, and reproduction in any medium, provided the original work is properly cited.
} 